Meta

Journal des tradlucteurs

Translators' Journal

\title{
Les premiers traducteurs (1760 à 1791)
}

\section{Paul A. Horguelin}

Volume 22, numéro 1, mars 1977

Histoire de la traduction au Canada

URI : https://id.erudit.org/iderudit/003101ar

DOI : https://doi.org/10.7202/003101ar

Aller au sommaire du numéro

\section{Éditeur(s)}

Les Presses de l'Université de Montréal

\section{ISSN}

0026-0452 (imprimé)

1492-1421 (numérique)

Découvrir la revue

Citer cet article

Horguelin, P. A. (1977). Les premiers traducteurs (1760 à 1791). Meta, 22(1),

15-25. https://doi.org/10.7202/003101ar d'utilisation que vous pouvez consulter en ligne.

https://apropos.erudit.org/fr/usagers/politique-dutilisation/ 


\section{Les premiers traducteurs (1760 à 1791)}

\section{LES SECRÉTAIRES BILINGUES SOUS LE REGIME PROVISOIRE (1760-1764)}

Si l'on peut faire remonter à Jacques Cartier l'histoire de l'interprétation au Canada, celle de la traduction ne commence véritablement qu'à la reddition de Québec, en 1759. Le changement de régime a en effet apporté, sur le plan linguistique, une nette transformation : l'interprétation entre colons francophones et indigènes est reléguée au second rang; c'est la traduction, de l'anglais au français et vice versa, qui prend la première place.

L'un des premiers problèmes que durent résoudre les nouveaux occupants fut celui de la communication. $\AA$ vrai dire, ils n'eurent guère le choix, car personne dans la colonie ne parlait anglais. On peut supposer que quelques traitants et coureurs des bois avaient acquis des notions de cette langue au cours de leurs échanges commerciaux avec les marchands d'Albany et de Boston, mais la masse de la population ne connaissait que sa langue maternelle. Il ne pouvait donc être question de lui imposer subitement une langue étrangère et, à défaut de stipulation précise dans les capitulations ou d'instructions venant de Londres, c'est l'état de fait qui dicta la conduite des gouverneurs britanniques.

Chose étrange, les capitulations de Québec et de Montréal ne contenaient, en effet, aucune disposition relative à la langue. Benjamin Sulte note à cet égard :

Quand Vaudreuil et Lévis dictaient la capitulation de Montréal [...] ils oubliaient d'y stipuler la protection de la langue française et, par là-même, ils ajoutaient aux éléments de faiblesse qui nous avaient fait succomber ${ }^{1}$.

Comment expliquer cet « oubli $\$$ ? Les historiens ne semblent pas avoir tenté de le faire. Ils se contentent de constater que "parler au peuple, du jour au lendemain, une autre langue que la sienne s'avérait tentative assez chimérique ${ }^{2} \gg$ et que «sous le régime militaire, la langue française bénéficia d'un statut presque officiel $^{s} \gg$.

Pendant quatre ans, le français allait ainsi être, en pratique, la langue officielle de la colonie, ce qui supposait évidemment le recours à la traduction. Dans une

1. Benjamin Sulte, la Langue française au Canada, Lévis, P.-G. Roy, 1898.

2. Lionel Groulx, Histoire du Canada français, Montréal, Fides, 1960, vol. II, p. 16.

3. Michel Brunet, les Canadiens après la conquête, Montréal, Fides, 1969, p. 24. 
première étape, le bilinguisme naissant se manifestera surtout dans deux domaines : les proclamations officielles et l'administration de la justice. Ce n'est qu'après le traité de Paris qu'il s'étendra à un troisième secteur, celui du commerce.

Avant de quitter la colonie pour aller établir son quartier général à New York, en septembre 1760, Amherst avait maintenu Murray à la tête du gouvernement de Québec, nommé Burton à Trois-Rivières et Gage à Montréal. Ces trois gouverneurs s'étaient adjoint un officier bilingue à titre de secrétaire et, ironie ou vengeance de l'Histoire, leur choix s'était porté sur trois descendants de huguenots ayant quitté la France à la révocation de 1'édit de Nantes, en 1685. C'est ainsi que les proclamations des gouverneurs furent rédigées ou traduites par des officiers britanniques au non bien français : Cramahé à Cuébec, Bruyères à Trois-Rivières et Maturin à Montréal. Lorsqu'en 1763 le colonel Haldimand, britannique d'origine suisse, sera nommé gouverneur de Trois-Rivières, c'est à un de ses anciens compatriotes, Conrad Gugy, qu'il confiera le poste de secrétaire. Les hommes qui servirent d'intermédiaires entre les gouverneurs et la population étaient donc tous des britanniques de fraîche date, et c'est vraisemblablement dans leur famille qu'ils avaient appris le français.

On peut se demander ici quel fut le rôle des secrétaires aux côtés des gouverneurs et quelle part ils prirent à la rédaction ou à la traduction des «placards ", comme on appelait alors les proclamations officielles. Au moment où ils entrent en fonction, les secrétaires ont le grade de capitaine, sauf Maturin qui est lieutenant ; ce sont donc des officiers subalternes, mais leur poste les place dans une situation privilégiée du fait qu'ils évoluent dans l'entourage immédiat du gouverneur, rédigent sa correspondance, partagent ses secrets, transmettent ses ordres ou instructions aux classes dirigeantes et à la population. Cramahé, en particulier, est l'homme de confiance de Murray qui le chargera de missions délicates auprès du haut clergé canadien et des autorités de Londres. On ne dispose malheureusement que de peu de renseignements sur la façon dont ces secrétaires travaillaient et sur l'aide dont ils disposaient. Il est probable qu'une partie des proclamations, notamment les premières, ont été rédigées par eux à partir de directives générales fournies par les gouverneurs; ce n'était donc pas, à proprement parler, des traductions. On peut supposer aussi que des Canadiens collaborèrent à l'établissement des textes, ce qui expliquerait la présence de quelques mots du terroir dans les proclamations : par exemple, "dans les côtes » pour « à la campagne ». Mais cette collaboration ne put intervenir, au début, qu'au stade de la révision et non de la traduction, car en 1760 aucun Canadien n'avait une connaissance suffisante de l'anglais pour traduire le texte des proclamations. Bien qu'on ne puisse établir avec certitude si les secrétaires ont rédigé ou traduit les proclamations, seuls ou avec l'aide de Canadiens, il est clair qu'ils en ont été les principaux auteurs et que les textes publiés sous leur signature avaient reçu leur approbation. Étant donné que pendant les quatre années du régime militaire, les secrétaires vont être les principaux agents du bilinguisme officiel, et que les proclamations seront les seuls documents officiels rédigés en français, si l'on exclut la correspondance des gou- 
verneurs, la biographie de ces secrétaires et l'examen de leurs proclamations revêtent, pour notre propos, un intérêt primordial.

Le plus illustre des quatre secrétaires qui vont retenir notre attention est sans conteste Hector Théophilus Cramahé ${ }^{4}$. Alors que ses trois confrères termineront une carrière sans éclat dans l'armée, Cramahé accédera aux plus hautes fonctions dans l'administration de la colonie. Né en 1721 dans une famille de huguenots français réfugiés en Angleterre, il s'engage dans l'armée à dix-neuf ans et, après avoir servi aux Antilles, en Flandre et dans deux expéditions contre la France, est promu capitaine en 1754. Il prend part aux sièges de Louisbourg et de Québec, et c'est le 2 novembre 1760 que Murray le choisit officiellement comme secrétaire. L'année suivante, son régiment est envoyé dans les colonies australes ; à la demande de Murray qui désire le garder à son service, Amherst l'autorise à vendre sa compagnie pour 1400 louis. En 1764, Cramahé espère obtenir le poste de gouverneur de Montréal, avec l'appui de Murray, mais le gouvernement de Montréal est aboli à l'établissement du régime civil. Murray l'envoie alors en mission secrète à Londres pour y exposer ses vues sur l'administration de la colonie. Il y restera jusqu'en 1766, année où il revient au Canada à titre de secrétaire du nouveau gouverneur, Guy Carleton. Nommé suppléant du receveur général en 1767, Cramahé se voit confier le poste de gouverneur intérimaire de la province de Québec en 1770; il devient président du Conseil législatif en 1775, puis membre du Conseil privé, malgré l'opposition du juge en chef Peter Livius qui le trouvait trop sympathique à la cause des Canadiens. Ne pouvant s'entendre avec le gouverneur Haldimand, qui avait succédé à Carleton, Cramahé démissionne en 1782. Il est nommé lieutenant-gouverneur de Détroit en 1785 et meurt à Londres le 8 juin 1788.

A Montréal, le gouverneur Thomas Gage a pris pour secrétaire Gabriel Maturin ${ }^{5}$, irlandais de naissance et, lui aussi, descendant de huguenots français. Lieutenant au $35^{\mathrm{e}}$ régiment d'infanterie, Maturin avait combattu à Louisbourg avant de prendre part au siège de Québec. Blessé à la bataille des Plaines d'Abraham, il suit néanmoins son régiment à Montréal où il reçoit sa nomination de secrétaire, le 22 septembre 1760 . Lorsque Gage est nommé au commandement des troupes de New York, en octobre 1763, son secrétaire l'accompagne. L'année suivante, Maturin est promu capitaine et il reste attaché au général Gage jusqu'en 1756. Il est muté au $31^{\text {e }}$ régiment d'infanterie en 1767 , puis on le retrouve à Montréal en 1773, au service de l'Intendance, et au mois de mai de l'année suivante, il est nommé « assistant député quartier-maître général » dans la même ville. Le 21 juillet 1774, Maturin est secrétaire du commandant en chef à Salem, au Massachusetts. Son nom cesse de figurer sur les rôles de l'armée en 1775 .

John des Bruyères ${ }^{6}$, qui au Canada supprime la particule de son nom, était issu d'une famille noble dont plusieurs membres s'étaient distingués au service de

4. Cf. Le Jeune, R.P., Dictionnaire général de biographie, Ottawa, 1931, vol. I, p. 447.

5. Cf. Francis-J. Audet, "Gabriel Maturin 》, in Bulletin de recherches historiques, vol. 31, $\mathbf{n}^{0} 9$, septembre 1925, p. 350.

6. Cf. Francis-J. Audet, op. cit., p. 342-343. 
la France. Cet autre descendant de réfugiés huguenots était entré dans l'armée anglaise, dans le mème régiment que Maturin, quelques mois seulement avant de prendre part au siège de Québec. Le 15 septembre 1759, deux jours après la bataille des Plaines d'Abraham, il est désigné comme «secrétaire auquel tous les prisonniers et les papiers pris à l'ennemi seront confiés ». En garnison à Québec, il écrit au général Townshend une lettre datée du 19 mai 1760 dans laquelle il relate les événements qu'il vient de vivre. Il est présent à la capitulation de Montréal et, en octobre 1760, devient secrétaire du gouverneur de Trois-Rivières, le colonel Ralph Burton. Lorsque celui-ci se rend à La Havane, en mai 1762, Bruyères remplit les mêmes fonctions auprès de Haldimand, gouverneur par intérim. Il retrouve son ancien chef en mars 1763 et, quand celui-ci est nommé gouverneur de Montréal en octobre de la même antiée, il le suit dans cette ville. Le 11 août 1764, soit le lendemain de l'instauration du gouvernement civil qui entraînait la suppression de son poste, Bruyères achète à Pierre Méziers une maison voisine de celle de $\mathbf{M}$. de Vaudreuil, à Montréal, et qu'un incendie devait détruire en 1768. Par son mariage avec Catherine Pommereau, fille de François Pommereau et de Françoise Boucher de Boucherville, il était devenu propriétaire d'une partie de la seigneurie de Bécancourt et il en acheta une autre partie de sa belle-sœur, Reine Pommereau. Bien que cette acquisition l'eût rendu maître de la moitié de la seigneurie, il chercha par la suite à augmenter son domaine : en juillet 1771 , il s'adressait à cette fin à son ancien collègue Cramahé et, en septembre 1784, alors qu'il était en Angleterre, son notaire renouvelait la demande au gouverneur Haldimand, apparemment sans succès. On ne connaît pas la date exacte de la mort de John Bruyères, mais on sait qu'elie est antérieure au 18 janvier 1787, comme en fait foi une pétition de son fils Ralph Henry.

Lorsque le colonel Haldimand devint gouverneur de Trois-Rivières en octobre 1763, il choisit, pour remplacer Bruyères quii suivait Burton à Montréal, un ancien officier d'origine suisse : Conrad Gugy ${ }^{7}$. Appartenant à une famille de militaires, Gugy, né en 1734, avait d'abord servi en Hollande avant de s'engager dans l'armée anglaise. Capitaine sous les ordres de Wolfe à Québec, il décida bientôt de quitter l'armée et de s'établir au pays. Nommé secrétaire en 1763, il remplit ensuite les mêmes fơnctions aux conseils législatif et exécutif. Il mourut célibataire, à Yamachiche, le 10 avril 1786. Son frère Barthélemy, colonel des Gardes suisses en France au moment de la Révolution, passa en Angleterre avec son fils Louis, lieutenant au même régiment, et tous deux s'établirent par la suite au Canada avec leur famille.

Après ces quelques notes biographiques sur les secrétaires bilingues, il peut être intéressant d'examiner la qualité des textes qu'ils ont rédigés ou traduits. Les proclamations des gouverneurs, sous le régime provisoire, ont été publiées dans le Rapport de l'archiviste du Canada (1918) ; elles font aussi l'objet de l'une des rares études sur l'histoire de la traduction au Canada, que nous devons à Pierre Daviault ${ }^{8}$. C'est à ces sources que nous puiserons pour faire une brève analyse de la langue des secrétaires bilingues.

7. Cf. Le Jeune, R.P., op. cit., p. 723.

8. Pierre Daviault, "Traducteurs et traduction au Canada », in Mémoires de la Société royale du Canada, tome $38,1944$. 
Très riches en renseignements d'ordre historique, les proclamations ne sont pas moins instructives sur le plan linguistique. La grande difficulté qui se présente, toutefois, pour le lecteur $\mathrm{du} \mathrm{xx}^{\mathrm{e}}$ siècle, est de faire la part entre les tournures et termes devenus archaïques, d'une part, et les erreurs de traduction proprement dites - maladresses, calques et anglicismes -, d'autre part. Les exemples de cette confusion possible abondent; citons-en quelques-uns : "La Rivière de Saint-Laurent \$ (que l'on retrouve sur des cartes françaises de l'époque), «marchandises sèches \$ (encore taxée d'anglicisme de nos jours, cette expression est pourtant attestée par l'encyclopédie Universalis), "commission d'officier \$ (au lieu de «brevet», mais les commissions existaient sous les rois de France). On relève aussi quelques canadianismes : "traîne 》 (sley), "cajeux 》 (raft), «piastre \$ (dollar). Une autre particularité est la tendance à la francisation : \& le gouverneur Jacques Murray » ou même «M. de Murray», «S.E. monsieur le chevalier Amherst ». Les secrétaires bilingues n'ont certes pas évité tous les pièges de la traduction : il est difficile d'excuser " aux heures de son office 》 (his office hours), «député juge avocat», "en échange pour des marchandises ; mais à côté de quelques défaillances, combien d'expressions heureuses rendues qu'oublieront les traducteurs des siècles suivants : « sous huit jours 》 (et non «en dedans »), «autant qu'il vous a été possible» (et non «en autant que»), "vendre des boissons » (non « des breuvages »). À cet égard, Pierre Daviault note un fait significatif : alors qu'on lit «tarif des voitures \$ dans le texte d'une proclamation, le traducteur de l'index du Rapport de l'archiviste écrit, en 1918 : « Taux des véhicules $\gg$ !

En somme, le français des proclamations, à part quelques interférences, est de bonne venue. On peut lui reprocher de «sentir le terroir \$ (mais est-ce un reproche ?), certainement pas d'être une copie servile de l'anglais. Voici, à titre d'exemple, un extrait d'une proclamation de Murray :

Apres une Campagne rude et penible, Nous ne Pensions qu'a donner du Repos aux Troupes et laisser Respirer le Peuple en tranquillité, apres les Malheurs qu'il a Essuyés pendant le Cours de cette Année, Marquée par tant d'Evenemens Grands et decisifs - Mais Malgré des Intentions si Humaines, Je me vois rappelé en Campagne par la Fidélité que je dois a mon Prince, et Pour Proteger le Peuple Soumis a Ses Armes -

On se prend à souhaiter que toutes les traductions publiées de nos jours soient rédigées dans une langue aussi châtiée !

\section{VERS LE BILINGUISME (1764-1768)}

À la fin d'août 1764, l'administration militaire fait place à un régime civil. Les gouvernements de Montréal et de Trois-Rivières sont abolis, et Murray devient le premier gouverneur de la «province de Québec». Dorénavant, les proclamations émaneront de Québec et seront publiées dans la Gazette de Québec.

Cette nouvelle période est marquée par une nette détérioration de la qualité des "placards». La rédaction parallèle, dont on commence à reparler à notre époque, cède la place à la traduction proprement dite. En effet, si Cramahé n'a 
pas encore le titre de traducteur, il traduit et ne rédige plus les proclamations. Or, très rapidement, ses textes vont regorger d'anglicismes et de calques, dont Daviault fait un relevé édifiant : "rivières qui versent (empty) dans le SaintLaurent », " tribus avec lesquelles il a connexion (he is connected) 》, " ne fassent aucun trafic avec, ni ne vende aux sauvages》, «je fais sortir ( $I$ issue) cette proclamation », « le roi français », " offenseurs (offenders), etc. Le contraste est si frappant qu'on peut se demander si le traducteur Cramahé est bien le même homme que le secrétaire bilingue... Il y a là une énigme à résoudre : ou bien les dernières traductions signées par Cramahé ne sont pas de sa plume, ou bien notre traducteur a été la victime d'une anglicisation aiguë et rapide ! Notons aussi que Cramahé, comme d'autres traducteurs de cette époque, laisse en anglais les termes dont il ignore l'équivalent ou bien fait suivre la traduction du mot anglais entre parenthèses : « mandat » (warrant).

La traduction «officielle» n'est pas la seule, hélas, à donner un mauvais départ au bilinguisme. Le 21 juin 1764 paraissait le premier numéro de The Quebec Gazette / la Gazette de Québec, ancêtre des journaux canadiens. Ses deux fondateurs, William Brown et Thomas Gilmore, imprimeurs venus de Philadelphie, étaient sans doute animés de bonnes intentions, mais ils allaient avoir le douteux honneur de susciter une nouvelle «profession», celle de traducteur improvisé, et de créer - avant le mot - une nouvelle langue : le franglais. Ainsi, quatre ans seulement après le chargement de régime, la traduction au Canada s'engageait dans une voie sans issue et commençait à mériter l'opprobre qui restera attachée, sans distinction, à cette activité.

Dans un article ${ }^{9}$ où il fait 1'historique du "premier journal bilingue en Amérique du Nord », Antonio Drolet, bibliothécaire en chef aux Archives du Québec, s'est amusé à relever les perles qui émaillent les colonnes de la Gazette. Il note, comme nous l'avons fait plus haut, la tendance à la francisation des noms propres : William devient Guillaume, le Brig Naney se métamorphose en «brigantin la Nannon ». Le vocabulaire spécialisé est évidemment malmené : les Court sticking plasters sont «des emplâtres collantes de la Cour», et les tire-lait (breast glasses), des « verres à mamelles \$! Les anglicismes et les barbarismes font bon ménage dans une autre série d'expressions : «les connexions réciproques des puissances de l'Europe », "les occurrences matérielles des provinces», « le fait avait arrivé par accident», «le cap du Tourment» (cap Tourmente), \&gens élevés dès le bas âge à mépriser les tempêtes », "il arriva hier un exprès de Montréal, avec la pacquet de la Nouvelle-Angleterre » (on retrouve ici l'ancêtre du «paquebot $>$ - packet-boat).

L'auteur, qui n'est pas tendre pour les traducteurs ( $\ll$ les bons traducteurs sont toujours moins nombreux qu'ils ne le pensent eux-mêmes $\gg$ ), reconnaît quand même qu'il s'agissait de novices et se demande s'ils avaient été recrutés par cette annonce parue dans la Gazette : «... un jeune homme d'esprit, d'environ quatorze ans, qui peut procurer de bonnes recommandations, et peut se faire entendre en Anglois et en François, il sera d'autant plus agréable. »

9. Antonio Drolet, «Le premier journal bilingue en Amérique du Nord », in Columbia, New Haven (Conn.), mars 1971, p. 42-43. 
Cette expérience de journalisme bilingue sera de courte durée. En effet, si la Gazette de Québec continua de paraitre jusqu'en 1874, elle devint unilingue anglaise quelques années après sa fondation.

\section{FRANÇOIS-JOSEPH CUGNET, PREMIER TRADUCTEUR OFFICIEL (1768-1789)}

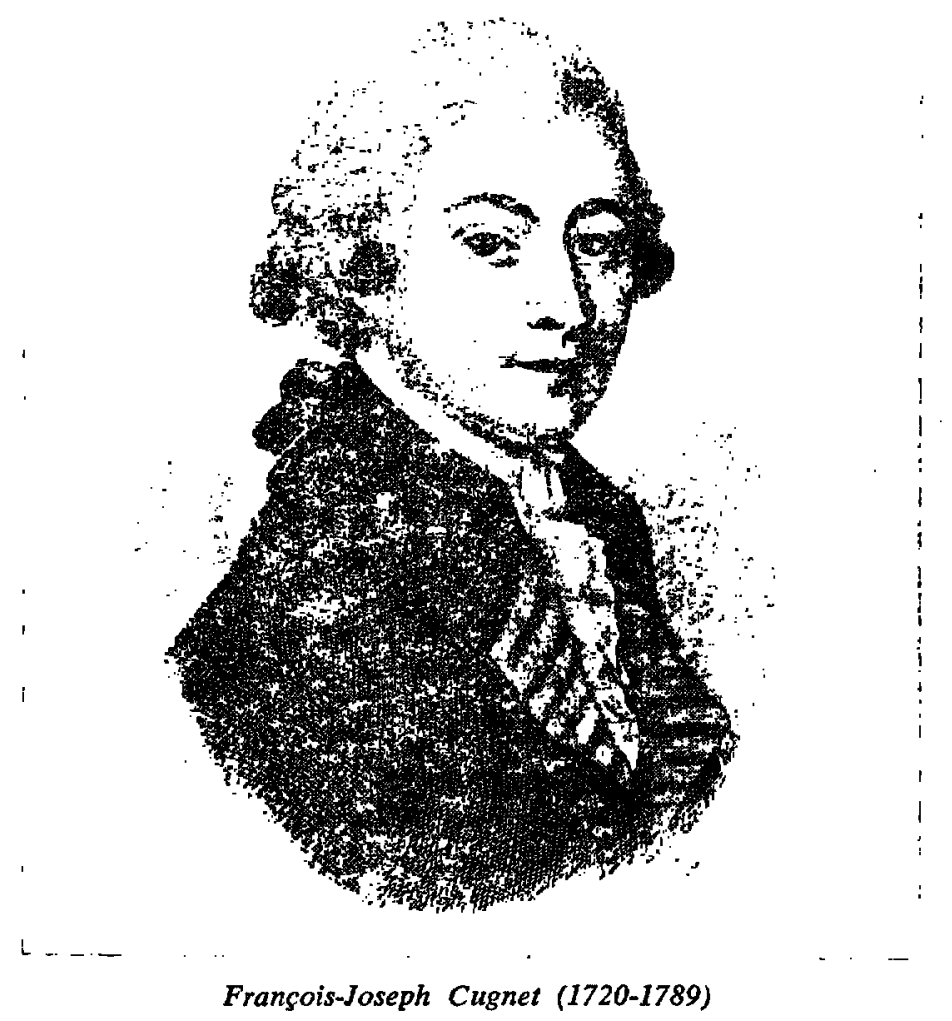

En 1763, une Proclamation royale avait supprimé les lois françaises, mais les gouverneurs britanniques constatèrent rapidement qu'il était impossible d'administrer la justice avec un corps de magistrats qui ne parlaient pas la langue de la population et, ignorant le droit français, ne pouvaient interpréter les actes passés sous l'ancien régime. L'avènement du gouvernement civil avait aussi renforcé l'appareil administratif et multiplié les occasions de communiquer avec les « nouveaux sujets », comme on appslait les Canadiens de souche française. Lorsque Carleton vint remplacer Murray en 1767, il se trouva donc aux prises avec un grave problème de traduction. D'une part, il estimait essentiel de faire recenser et traduire les lois et ordonnances françaises, les magistrats anglais s'en déclarant incapables; d'autre part, il avait besoin d'un «secrétaire français 》 pour assurer la traduction des proclamations et autres textes officiels. Or, à sa connaissance, il n'y avait qu'un seul Canadien apte à remplir ces tâches : François-Joseph Cugnet. 
Le 24 février 1768, Cugnet était nommé « French Translator » et « Secretary to the Governor and Council ». La veille, le Conseil avait décidé que «such a good and sufficient translator shall have an appointment of 5 shillings sterling per day».

Curieux personnage, à vrai dire, que cet homme qui, pendant plus de vingt années, allait assurer la traduction officielle dans la province de Québec. Dans l'étude biographique qu'elle lui a consacrée ${ }^{10}$, et dont nous sommes largement redevables, Marine Leland n'hésite pas à écrire qu'il «n'existe pas de figure plus étrange dans l'histoire du Canada français... »

Né à Québec en 1720, François-Joseph Cugnet appartient à une famille de juristes français. Son père, François-Étienne, était directeur général du Domaine du roi. Homme cultivé (sa bibliothèque de 1500 ouvrages n'avait guère d'égal en Nouvelle-France), il se lança dans plusieurs aventures commerciales, dont la direction des Forges de Saint-Maurice, qui le conduisirent à la ruine. $\grave{A}$ ses soucis d'argent vinrent bientôt s'ajouter les inquiétudes que lui causait son fils aîné. Après des études à Québec, où il assiste notamment aux conférences de droit du procureur général Guillaume Verdier, François-Joseph travaille quelque temps dans le bureau de son père, puis disparaît. On le retrouve en 1751 dans une prison de La Rochelle, à la suite d'une affaire de duel. Dans son interrogatoire, il déclare être "ancien écrivain de la Marine à Saint-Domingue, [...] demeurant ordinairement dans la Principauté d'Orange ». Ayant appris la mort de son père, il rentre à Québec en 1752 , après avoir contracté une dette que sa mère devra rembourser. Une charge de conseiller-assesseur lui est refusée, et il entre comme "aide » à la direction du Domaine. En 1757, il épouse Marie-Josèphe de La Fontaine de Belcour, nièce de Louis Jolliet, âgée de dix-sept ans.

La longue carrière de Cugnet au service de l'administration britannique commence à la reddition de Québec... en même temps qu'un mystère qui jette des doutes sur son rôle. Bien qu'ayant pris part à la défense de la ville, Cugnet ne suit pas l'armée qui se replie sur Montréal. Plus tard, on l'accusera d'avoir indiqué à Wolfe le sentier qui mène de l'Anse-au-Foulon aux Plaines d'Abraham, ce qui lui vaudra d'être appelé « le traître Cugnet 》. L'accusation n'a jamais été prouvée, mais l'on sait que Vaudreuil et Bigot instituèrent une enquête sur son compte, interrompue par la capitulation de Montréal. Cugnet eut à se défendre d'une autre accusation : nommé par Murray « juge-administrateur des paroisses de Charlesbourg, de Beauport et de la Petite-Rivière », il semble s'être acquitté de sa tâche avec un zèle que n'apprécièrent pas ses administrés, si l'on en juge par une requête qu'il présenta au Conseil en vue de «se justifier de touttes les Calomnies débitées contre luy ». Murray lui garda néanmoins sa confiance, puisqu'il le nomma «procureur général de la côte nord du district de Québec » en 1760 et «grand-voyer du district de Québec » en 1765.

A l'arrivée de Carleton, successeur de Murray, Cugnet se lie immédiatement d'amitié avec le nouveau gouverneur. Il fait aussi la connaissance du nouveau procureur général, Francis Masères, qui allait devenir son grand ennemi. Autre

10. Marine Leland, * François-Joseph Cugnet (1720-1789) \$, in la Revue de l'Université Laval, vol, XVI, no 1 (septembre 1961) à vol. XXI, no 4 (décembre 1966). 
descendant de huguenots réfugiés en Angleterre, Masères honnit tout ce qui est français et catholique. Les deux hommes ne vont pas tarder à se heurter sur la question du régime juridique de la colonie, entamant une longue polémique qui se poursuivra après le retour de Masères en Angleterre. C'est dans cette lutte que Cugnet fera figure de "champion des lois françaises ». En fait, il semble qu'il défendait plutôt ses intérêts : héritier par sa femme de la seigneurie de Mingan, c'est surtout pour protéger son bien qu'il défendait la tenure seigneuriale du code civil français. Son attitude sera aussi équivoque lorsqu'il se fera l'ardent défenseur d'une «Chambre d'assemblée ». S'il rédige des pétitions où il se prétend le porte-parole des «Habitans de la Province de Québec», s'il en signe d'autres en s'identifiant comme un "patriote canadien », c'est aussi lui qui écrit à Blackstone : " Cette Chambre Est le seul moïen qui puisse rendre, ainsi qu'il le faut nécessairement dans quelques années, les Canadiens Vrais anglais, \& les engager d'En adopter les loix. 》Mais revenons à notre traducteur.

Les premières ordonnances traduites par Cugnet après sa nomination paraissent sans sa signature. Il ne signera ses traductions qu'à partir du 11 novembre 1768 : «F.-J. Cugnet, s.f.» (secrétaire français). Que valent ces traductions? Citons le jugement de Pierre Daviault avant d'en donner un échantillon : * Cugnet... était un bon traducteur moyen. J'entends par là que, sans écrire un français nettement abâtardi (il s'était formé sous le régime français), il a un style qui se ressent de l'influence de l'anglais. C'est déjà, dans la tournure de la phrase, la naissance de cette langue de traduction qui est maintenant notre plaie. $\gg \mathrm{Et}$ Daviault relève les erreurs suivantes : «ordre en conseil (Order in Council), « rendre dociles à justice » (render amenable to justice), « une partie coupable » (a guilty party), «toutes telles personnes (all such persons), «cité 》 (city), " ordonnance ci-đessus récitée (ordinance above recited) ${ }^{11}$. Voici maintenant une traduction de 1768 qui fait mieux ressortir le style de Cugnet :

\section{AN ORDINANCE FOR PREVENTING ACCIDENTS BY FIRE}

Whereas many Houses in the Towns of Quebec and Montreal in this Province have of late taken. Fire, and some of them have been destroyed thereby, which has been owing for the most Part to the Negligence of the Inkabitants of the said Houses, in not causing the Chimneys thereof to be swept so often as they should have been, and by keeping Ashes in the said Houses on wooden Floors or in wooden Vessels, and other such Instances of Carelessness; in Order therefore to prevent such unhappy Accidents for the Time to come, and to increase the Means of extinguishing Fires in Houses when they do happen, It is Ordained by the LieutenantGovernor of this Province, by and with the Advice and Consent of the Council of the sane...

\section{ORDONNANCE POUR PREVENIR LES INCENDIES}

$\mathrm{Vu}$ que plusieurs maisons dans les Villes de Québec et de Montréal, en cette province, ont depuis peu pris feu, et que quelques-unes d'icelles ont été consumées, ce qui a été pour la majeure partie l'effet de la négligence des habitants des dites maisons, en ne faisant pas ramonner les dites cheminées aussi souvent qu'elles auroient dû l'être, et en conservant des cendres dans les dites maisons sur des planchers ou des vaisseaux de bois, et par d'autres semblables exemples de négligence : C'est pourquoi à fin de prévenir de pareils accidents facheux pour l'avenir, et pour augmenter les moyens d'éteindre le feu dans les maisons quand ce malheur arrivera, Il est Ordonne, par le Lieutenant-Gouverneur de cette Province, par et avec l'avis et le Consentement du Conseil d'icelle...

11. Pierre Daviault, op. cit., p. 83. 
Cugnet, qui a une assez haute opinion de lui-même, n'hésite pas à critiquer la prose de ses confrères, comme en témoigne cette lettre adressée en 1773 aux imprimeurs de la Gazette :

\section{Messieurs}

Comme vous avés inséré dans votre dernière Gazette à l'article de Londres, des débats qui se sont élevés en la Chambre des Communes, à l'occasion des affaires de cette Province, dont la Traduction française n'est point correcte (sans doute parceque Vous n'avés pas donné au Traducteur le tems de les travailler pour les rendre dans toutes leurs forces dans l'Idiome de la langue française) on Vous en adresse la traduction suivante qui est intelligible...

Au lecteur d'en juger :

\section{TEXTE ORIGINAL}

The English laws are introduced among them [les Canadiens], but for want of knowing what they are, the descent goes upon the maxim of the French laws; and then come a pack of rascals, of pettifoggers who go to the heirs according to our laws, and instigate them to lay in claims, which throw all in confusion, and in this manner, the people are fleeced and ruined.

\section{VERSION DE LA GAZETTE}

Les lois anglaises sont introduites parmi eux, mais faute de les connaitre, on y déroge en suivant les maximes des lois françaises, et ensuite vient une troupe de coquins, de charlatans, qui vont trouver les héritiers, suivant nos lois et les poussent à produire des prétentions, qui jettent tout dans la confusion, et le peuple est de cette manière dépouillé et ruiné.

\section{VERSION DE CUGNET}

Les lois Anglaises sont introduites parmi Eux, mais faute de les Connaître, on $\mathrm{y}$ succède suivant les maximes des loix françaises. Une bande d'Avocats ignorans \& de mauvaise foy s'insinuent $\mathrm{En}$ ce Paîs dans les familles, \& les Excitent à faire des Demandes en Justice, conformément à nos loix, ce qui produit une confusion qui entraîne avec Elle le malheur et la ruine du Peuple.

À partir de 1768, Cugnet s'affaire à la rédaction de quatre traités de droit français que publiera en 1775 William Brown, imprimeur de la Gazette. Ces publications, officieusement subventionnées par le gouverneur Carleton, ne pouvaient arriver à un meilleur moment, puisque l'Acte de Québec venait de rétablir le code civil français. Elles valurent à Cugnet la renommée de «jurisconsulte », bien qu'il se défende, dans ses préfaces, d'être expert en la matière.

En 1777, avant de quitter le Canada, Carleton nomme Cugnet « greffier du Papier Terrier» (cadastre) et avocat, titre que ce dernier s'était attribué dès 1771 ! En 1779, Cugnet participe à la rédaction des règlements de la « Communauté des avocats » (Barreau), dont son fils, Jacques.François, va être nommé secrétaire. Il est alors au sommet de sa carrière. Les fonctions qu'il cumule, si elles le tiennent très occupé, lui assurent un bon revenu : il reçoit $£ 110$ à titre de secrétaire français, $£ 90$ comme greffier du Papier Terrier, en plus de suppléments' appréciables pour les rapports qu'il rédige à la demande du gouverneur et les nombreuses consultations qu'il donne (une seule d'entre elles lui rapporte $£ 74)$. Pourtant, les dernières années de sa vie sont assombries par des soucis 
d'argent (il est dépensier comme l'était son père et comme le sera son fils) et des procès interminables qu'il perd régulièrement, même s'il n'hésite pas à falsifier des actes pour aider sa cause...

Épuisé par sa charge de travail, harcelé par ses créanciers, souffrant de la goutte, Cugnet avait demandé au gouverneur Haldimand de lui désigner un successeur en la personne de son fils Jacques-François, ce qui lui fut refusé. Lorsque son ami Carleton, devenu lord Dorchester, revint au Canada en 1788, il accéda à cette demande : Jacques-François exerce les fonctions de traducteur et de secrétaire conjointement avec son père pendant quelques mois, puis lui succède. Le 11 avril 1789, Cugnet traduit sa dernière ordonnance. Il meurt le 16 novembre.

Les traductions que signe Cugnet vers la fin de sa carrière contrastent par leur médiocrité avec sa production antérieure de traducteur et de juriste. Comme dans le cas de Cramahé, on peut supposer qu'elles sont l'œuvre d'autres traducteurs, soit son fils, soit Pierre-Amable de Bonne, avocat dont le nom apparaît sous quelques traductions officielles. C'est le même de Bonne qui assurera l'intérim pendant les nombreux * congés de maladie» de Jacques-François Cugnet : de santé délicate, celui-ci devait mourir en 1797 à l'âge de trente-neuf ans.

Après un bon départ, assuré par les secrétaires bilingues sous le régime provisoire, la traduction officielle s'est donc maintenue à un niveau très convenable pendant les vingt années où elle est demeurée la responsabilité d'un seul homme : François-Joseph Cugnet. On peut certes reprocher au «secrétaire français » de suivre trop fidèlement la structure anglaise, mais il ne faut pas oublier que la littéralité a été un critère d'excellence à plusieurs époques de l'histoire de la traduction. L'indulgence s'impose également dans le cas du vocabulaire juridique employé par Cugnet : faut-il lui tenir rigueur de n'avoir pas su traduire subpcena, non-pros, writ of appeal, Common-pleas, lorsqu'on sait la difficulté que présente, encore de nos jours, la recherche d'équivalences sémantiques entre deux systèmes juridiques très différents ? Pourtant, en cette fin du XvIIr siècle, on voit déjà s'amorcer une dégradation de la qualité des textes traduits : le charabia a fait son apparition dans les colonnes de la Gazette, le calque et l'anglicisme à outrance vont bientôt caractériser la traduction officielle ; c'est le règne de l'amateurisme qui commence.

Paul A. Horguelin 